\title{
Data-driven selection of the minimum-gradient support parameter in time-lapse focused electric imaging
}

\author{
Frederic Nguyen ${ }^{1}$, Andreas Kemna ${ }^{2}$, Tanguy Robert ${ }^{3}$, and Thomas Hermans ${ }^{4}$
}

\begin{abstract}
We have considered the problem of the choice of the minimum-gradient support (MGS) parameter in focused inversion for time-lapse (TL) electric resistivity tomography. Most existing approaches have relied either on an arbitrary choice of this parameter or one based on the prior information, such as the expected contrast in the TL image. We have decided to select the MGS parameter using a line search based on the value of the TL data root-mean-square misfit at the first iteration of the nonlinear inversion procedure. The latter was based on a Gauss-Newton scheme minimizing a regularized objective function in which the regularization functional was defined by the MGS functional. The regularization parameter was optimized to achieve a certain target level, following the Occam principles. We have validated our approach on a synthetic benchmark using a complex and heterogeneous model and determined its effectiveness on electric tomography TL data collected during a salt tracer experiment in fractured limestone. Our results have determined that the approach was successful in retrieving the focused anomaly and did not rely on prior information.
\end{abstract}

\section{INTRODUCTION}

Time-lapse (TL) electric resistivity tomography (ERT) is being increasingly used to monitor subsurface processes. In particular, TL measurements have been used to study groundwater flow and solute transport (Kemna et al., 2002; Doetsch et al., 2012), moisture content (Oberdörster et al., 2010), vadose zones (Koestel et al., 2008), permafrost (Krautblatter et al., 2010), infiltration (Slater et al., 2010), seawater intrusion (Ogilvy et al., 2009), and, more recently, geothermal systems (e.g., Hermans et al., 2015). Interpretations from TL surveys are generally drawn with respect to the changes in the system from a reference state. Given the nonlinear forward operator $f()$, which maps a model of resistivity, represented by a vector $\mathbf{m}^{k}$, to the observable data, resistance, or $\log$ resistance values, represented by a vector $\mathbf{d}^{k}$, we are interested in finding TL changes $\mathbf{m}^{k}-\mathbf{m}^{0}$ at all times $k$, such that

$$
f\left(\mathbf{m}^{k}\right)=\mathbf{d}^{k}+\mathbf{e}^{k}
$$

where the superscript $k=1,2, \ldots, K$ represents the time index, 0 refers to the reference or baseline state, and $k$ refers to the monitor state. The vector $\mathbf{e}^{k}$ accounts for the data noise and modeling errors typically due to inadequate parameterization and/or limitations in discretization. Although systematic errors may be canceled though the data difference approach, random errors, if fully independent, will increase by a factor of $\sqrt{2}$ when differenced.

Different inversion approaches have been proposed to invert TL data ranging from the subtraction of two separately inverted models (Ramirez and Lytle, 1986) to spatiotemporal regularization (Brooks et al., 1999; Day-Lewis et al., 2002; Kim et al. 2009; Karaoulis et al., 2011; Singha et al., 2015). Incorporation of temporal information or working with data variations improves TL imaging. Regularization (Tikhonov and Arsenin, 1977; Zhdanov, 2002) remains one of the most popular solutions to solve TL inverse problems having a large number of parameters. The inverse problem is then generally treated as an optimization problem in which a properly designed stabilizing functional is minimized, typically using a Gauss-Newton scheme, under the condition that the chi-squared measure of the data misfit $\Psi_{d}\left(\mathbf{d}^{k}, \mathbf{m}^{k}\right)=\sum_{i}^{N} \frac{\left|d_{i}^{k}-f_{i}^{k}(\mathbf{m})\right|^{2}}{\left|\varepsilon_{i}^{k}\right|^{2}}$, with $N$ data points and data error $\varepsilon_{i}$,

Manuscript received by the Editor 12 April 2015; revised manuscript received 25 June 2015; published online 29 January 2016.

${ }^{1}$ University of Liege, Argenco Department, Applied Geophysics, Liege, Belgium. E-mail: f.nguyen@ulg.ac.be.

${ }^{2}$ University of Bonn, Department of Geophysics, Steinmann-Institute, Bonn, Germany. E-mail: kemna@geo.uni-bonn.de.

${ }^{3}$ Aquale Sprl, Fernelmont, Belgium. E-mail: t.robert@aquale.com.

${ }^{4}$ Formerly University of Liege, Argenco Department, Applied Geophysics, Liege, Belgium and Fund for Scientific Research-FNRS, Brussels, Belgium; presently Stanford University, Department of Geological Sciences, Stanford, California, USA. E-mail: thomas.hermans@ulg.ac.be.

(C) 2016 Society of Exploration Geophysicists. All rights reserved. 
reaches an adequate level corresponding with the a priori degree of uncertainties of the data and the model as follows:

$$
\Psi\left(\mathbf{m}^{k}\right)=\Psi_{d}\left(\mathbf{d}^{k}, \mathbf{m}^{k}\right)+\lambda \Psi_{m}\left(\mathbf{m}^{k}\right),
$$

where $\lambda$ is the regularization parameter. The choice of the model functional $\Psi_{m}\left(\mathbf{m}^{k}\right)$ in the objective function shapes the characteristics of the inverted model. The widely used smoothness constraint is defined by the functional (de Groot-Hedlin and Constable, 1990) in the following equation:

$$
\Psi_{m}\left(\mathbf{m}^{k}\right)=\int\left\|\nabla\left(\mathbf{m}^{k}\right)\right\|^{2} d v
$$

The minimum-gradient support (MGS) functional (Portniaguine and Zhdanov, 1999) can be used to produce sharp images when needed (Robinson et al., 2015):

$$
\Psi_{m, \mathrm{MGS}}=\int \frac{\nabla\left(\mathbf{m}^{k}\right) \cdot \nabla\left(\mathbf{m}^{k}\right)}{\nabla\left(\mathbf{m}^{k}\right) \cdot \nabla\left(\mathbf{m}^{k}\right)+\beta^{2}} d v .
$$

Such a functional requires the definition of an additional parameter $\beta$. It is a critical parameter that controls the sharpness of the resulting image. One of the difficulties in applying this regularization remains the choice of $\beta$ in equation 4 , in addition to the choice of $\lambda$ in equation 2. Most approaches are based on one of the following: synthetic tests (Blaschek et al., 2008); stability requirements (Zhang et al., 2012); arbitrary choices, for example, related to machine precision (Portniaguine and Castagna, 2004; Vignoli et al., 2015); prior information (Fiandaca et al., 2015); the mean value of model parameter gradients (Rosas Carbajal et al., 2012); or by choosing a value in the neighborhood of the maximum curvature point of the model functional over a range of $\beta$ values for the reference model (Zhdanov and Tolstaya, 2004). To address possible trade-offs between the regularization parameter and the MGS parameter, approaches such as the generalized L-curve for joint parameter selection could also be used (Belge et al., 2002).

The use of MGS regularization to TL changes in ERT will not only limit the occurrences of temporal changes in electric resistivity but also restrict the variations of these changes inside the different spatial zones. It is therefore appealing when sharp changes are expected. There is a growing number of applications and developments of the MGS regularization for TL studies (Ajo-Franklin et al., 2007; Kim and Cho, 2011; Rosas Carbajal et al., 2012). However, similar to static applications, there are many different approaches to select the MGS parameter $\beta$. Such diversity hinders the spread of usage of the MGS functional for TL changes and impacts the robustness of the approach.

We here propose to select the MGS parameter $\beta$ by minimizing the TL data misfit during the first iteration of the inversion process. This approach has the advantages over existing methodologies to rely only on the data and not on the prior knowledge of model parameters except for model sharpness. It is therefore robust and flexible. We validate our approach on a synthetic case, and we demonstrate it on field data collected during a salt tracer test in fractured limestone using a background data set and one TL data set.

\section{INVERSION METHOD}

The code used here to invert the ERT data is CRTOMO (Kemna, 2000). It uses a Gauss-Newton scheme to minimize iteratively an equation of the form of equation 2, based on a finite-element (FE) forward solver. The inverted parameters $\mathbf{m}$ are the $\log$ values of the electric conductivities of the elements of the FE mesh, and the inverted data $\mathbf{d}$ are defined as the $\log$ values of the measured resistances. The different model constraints (e.g., equations 3 and 4) can be applied on the TL changes $\mathbf{m}^{k}-\mathbf{m}^{0}$. When working with TL data, the data misfit of equation 2 is defined in terms of the temporal data changes $\mathbf{d}^{k}-\mathbf{d}^{0}$ (LaBrecque and Yang, 2001) as follows:

$$
\Psi_{d}\left(\mathbf{d}_{K}, \mathbf{m}_{K}\right)=\left\|\mathbf{W}_{\mathbf{d}}\left[\left(\mathbf{d}^{k}-\mathbf{d}^{0}\right)-\left(f\left(\mathbf{m}^{k}\right)-f\left(\mathbf{m}^{0}\right)\right)\right]\right\|^{2},
$$

where $\mathbf{W}_{\mathbf{d}}$ is the data-weighting matrix. The initial regularization parameter $\lambda$ of equation 2 is estimated from the mean row sums of the matrix $\mathbf{J}^{T} \mathbf{W}_{d}^{T} \mathbf{W}_{d} \mathbf{J}$, where $\mathbf{J}$ is the Jacobian matrix to balance the role of data misfit and regularization when solving the resultant normal equations; $\lambda$ is then optimized at each iteration step to decrease the root-mean-square (rms) of the error-weighted data misfit $\varepsilon^{\mathrm{rms}}(\mathbf{m})=\sqrt{\Psi_{d} / N}$ for $N$ given data. The iteration process is stopped when the rms value of the error-weighted data misfit reaches the value of one, for a maximum possible value of $\lambda$. The latter aspect is important to actually fulfill the optimization criterion (model functional minimum, subject to fitting the data) and to ensure that all data are fit to the same level when comparing different inversion results of the same data set. Note that some authors found that differencing the data could be difficult to achieve in practice, in particular, if electrodes cannot stay in place (Miller et al., 2008). In such a case, the minimum support functional could still be used together with a standard data misfit instead of the one of equation 5.

The MGS parameter $\beta$ is estimated by selecting the one that minimizes the TL data misfit at the first iteration of the inversion process, whereas the regularization parameter is at its initial value, i.e., before optimization of $\lambda$. This is achieved by performing a line search. The selected MGS parameter $\beta$ is then kept constant in the iterative procedure. Although the inverse problem is nonlinear, we may reasonably assume that the background model is relatively close to the solution and that the problem is only weakly nonlinear. Estimating the MGS parameter $\beta$ during the first iteration can therefore be justified by the TL nature of the data set and inversion. It is also a very convenient procedure computationally.

\section{VALIDATION ON NUMERICAL MODEL}

The numerical benchmark corresponds to bedrock overlaid by a loamy layer (Figure 1a). The FE model has 2430 rectangular elements. The reference state has a heterogeneous conductivity distribution (the bedrock ranges from 100 to $300 \Omega \mathrm{m}$, and the top layer ranges from 10 to $60 \Omega \mathrm{m}$ ), as will generally be the case in field applications, and the topography is also included. We seek to recover a local anomaly in resistivity $(-50 \%)$ that mimics a salt tracer that flows through a fracture network (Robert et al., 2012). The anomaly is sharp, slightly dipping, and focused $(3 \mathrm{~m}$ wide and $9 \mathrm{~m}$ high) at a depth of $10 \mathrm{~m}$ and extends downwards to approximately $20 \mathrm{~m}$ (Figure 1c). The electrode setup consists of 72 electrodes at the surface with spacing of $3 \mathrm{~m}$ between adjacent electrodes (the length of the electrode layout is $213 \mathrm{~m}$ ). The simulated data 
acquisition scheme is dipole-dipole with a maximum $n$ factor (dipole separation in units of dipole lengths) of six and an $a$ factor (dipole length in units of electrode spacing) ranging from one to nine leading to 1225 data points altogether. For the inversion, we use a uniform data weight (constant data error of 0.1 , corresponding to a $0.1 \%$ resistance error) in equation 5 and uncontaminated data.

Smoothness-constraint inversion results for the reference state and TL changes produce the results shown in Figure $1 \mathrm{~b}$ and 1d. All inversions converged on the basis of the criteria as detailed above. As can be seen from the reference model, the inversion results allow detection of the two layers but fails to recover the small resistivity contrast within the bedrock in which the preferential pathways are located. In addition, the topography of the bedrock is not perfectly delineated. For the TL results, the anomaly due to the conductive synthetic salt tracer is detected, but the size is largely overestimated due to the regularization and optimization of $\lambda$, and it is not possible to recognize the true shape of the anomaly, i.e., the dip of the fracture zone. In addition, the resistivity contrast of the anomaly is underestimated by one order of magnitude. Otherwise, there are few artifacts in the TL changes due to the smoothness constraint applied to the model changes and because the data are not overfitted. velange, Belgium. The objective of the ERT monitoring was to detect preferential flow paths in a fractured/karstified aquifer (Figure 2a and 2b) at a moderate depth (up to $20 \mathrm{~m}$ ). A detailed description is given by Robert et al. (2012). To characterize the fracture zone, a salt solution $(\mathrm{NaCl}$, at $154 \mathrm{~g} / \mathrm{l})$ was injected for $4 \mathrm{~h}$ $(500 \mathrm{l} / \mathrm{h})$ at a depth between 16.4 and $20 \mathrm{~m}$ using a well packer to constrain salt migration into a known fractured area (Figure $2 b$; see also Robert et al. [2011] for further details). The water table remained constant at a depth of $11.38 \mathrm{~m}$ during the experiment (Robert et al., 2012). The electric conductivity of groundwater before injection was measured in the well at approximately $0.52 \mathrm{mS} / \mathrm{cm}$. The injected solution containing the salt tracer had an electric conductivity of approximately $147 \mathrm{mS} / \mathrm{cm}$ for a salt concentration of $154 \mathrm{~g} / 1$ achieving a theoretical contrast ratio of 282 . Dilution in the well already lowered this ratio to approximately 150 .

The monitoring ERT line was composed of 72 electrodes spaced by $3 \mathrm{~m}$ and located $15 \mathrm{~m}$ away from the injection well (Figure 2a). Robert et al. (2012) estimate the depth of investigation at approximately $30 \mathrm{~m}$. Measurements were carried out with a dipole-dipole configuration with $a$ ranging from one to nine electrode spacings (dipole length) and $n \leq 6$ (dipoles separation) using an IRIS SYSCAL PRO device. Data error was estimated using reciprocal

\section{MGS TL inversion}

The MGS TL inversions are performed by applying equation 4 to the TL changes $\mathbf{m}^{k}-\mathbf{m}^{0}$. The impacts of varying the MGS parameter on the resulting TL images are shown in Figure $1 \mathrm{f}-1 \mathrm{k}$. The selection of parameter $\beta$ is shown in Figure 1e. We note that for $\beta$ below $10^{-6}$, the inversion fails to converge, and for $\beta$ greater than one, the inversion converges towards the smoothness-constraint results (Figure 1d). The selected value of $\beta\left(10^{-4}\right.$, Figure $\left.1 \mathrm{~h}\right)$, corresponding to the minimum of the TL data misfit after the first iteration step, yields TL model changes that are closer to the benchmark (compare Figure 1c and $1 \mathrm{~h}$ ). Inversion artifacts remain present and are mainly related to overshooting in areas with higher contrast typical in geophysical imaging (LaBrecque et al., 1996; Friedel, 2003; Rosas Carbajal et al., 2012; Aster et al., 2013). However, these artifacts have a contrast of less than $5 \%$ and are smaller than for other $\beta$ values. The resistivity contrast of the recovered anomaly is within one order of magnitude $(-15 \%)$ but still underestimated $(-50 \%)$. This is a consequence of the limited resolution of ERT at this depth relative to the size of the anomaly. Qualitatively, the position and, importantly, shape of the anomaly are much better recovered.

\section{FIELD APPLICATION}

\section{Experimental setup}

We present the analysis and imaging results for data collected during a salt tracer test in Ha-
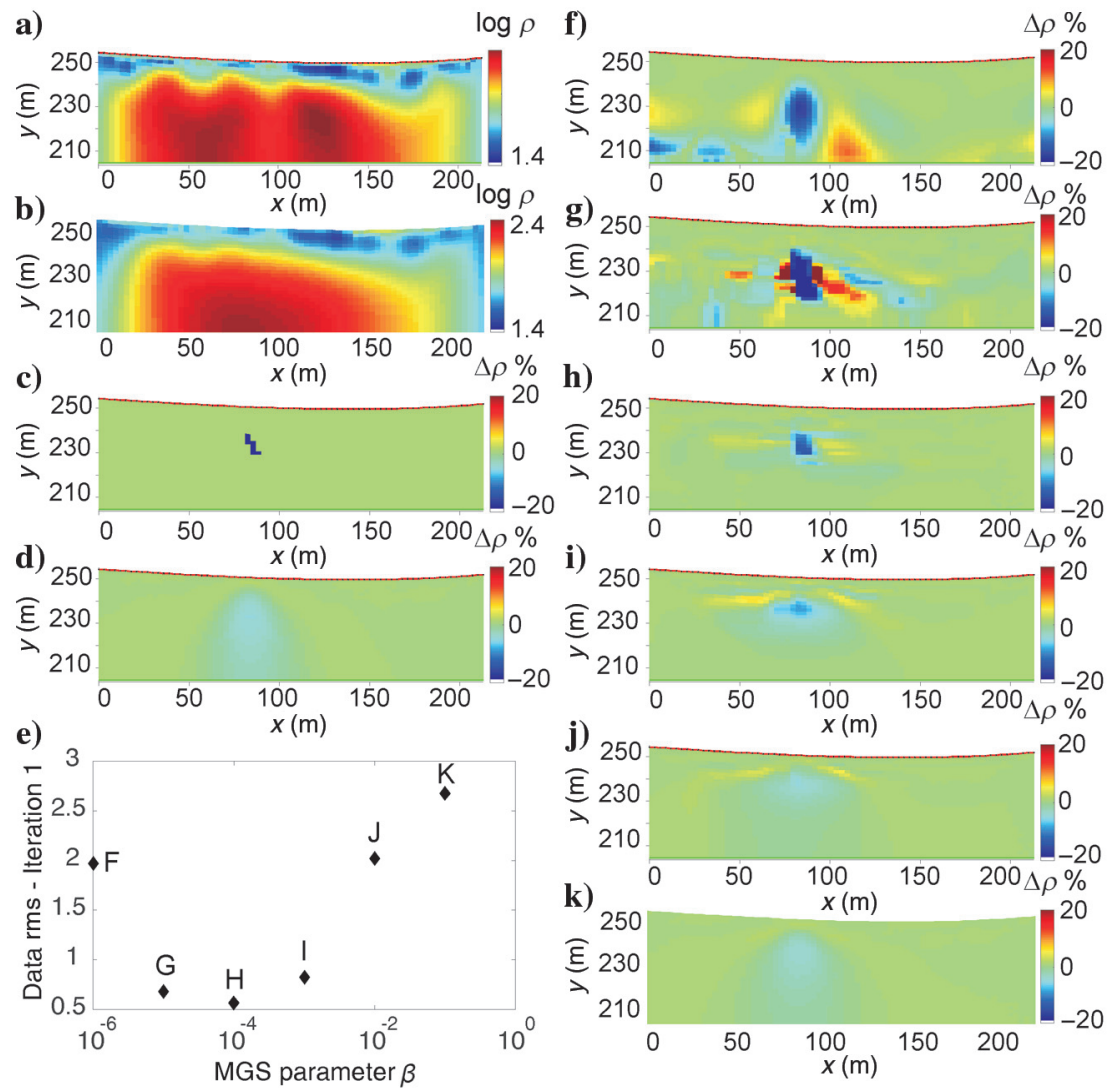

Figure 1 Synthetic modeling results. (a) Background model benchmark, (b) inverted background model with smoothness constraint, (c) TL model changes benchmark, (d) inverted TL model changes using smoothness constraint on the model changes, (e) selection of the MGS parameter $\beta$; the corresponding images (f-k) are indicated next to each point. Panels (f-k) show inverted TL model changes using MGS for different values of MGS parameters. Background model values are given as $\log _{10}$ of resistivity in $\Omega \mathrm{m}$ in panels ( $\mathrm{a}$ and $\mathrm{b}$ ), and TL model resistivity changes are given in percentage in panels (c, d, and $\mathrm{f}-\mathrm{k}$ ). 
measurements for the background state and after finishing the tracer test (Robert et al., 2012), and a uniform data weight (constant data error of 0.1 , corresponding to a $0.1 \%$ resistance error) was used for the TL data misfit in equation 5.

The reference image of profile P1 (Figure 2c) exhibits mainly two different layers. The top one corresponds to clayey loam with values up to $80 \Omega \mathrm{m}$. This unit composes a 5 to $10 \mathrm{~m}$ thick overburden and a weathered part of the limestone bedrock. The second layer below shows resistivity values from 100 to $320 \Omega$ m that reflect the saturated and fractured limestone bedrock. The result of the inversion of the TL data using a smoothness constraint on the TL model changes collected $8 \mathrm{~h}$ after injection displays a large negative anomaly $(-6 \%)$ (Figure 2d). Given the nature of the bedrock (Figure 2b), the diffuse nature of the inverted anomaly is considered to be primarily the result of the regularization, and a more adapted regularization scheme is therefore desired to fine-tune the results.

\section{MGS TL inversion}

The MGS TL inversion results are shown in Figure $2 \mathrm{f}-2 \mathrm{j}$ together with the selection of the corresponding MGS parameter $\beta$ (Figure $2 \mathrm{e}$ ). For values of less than $10^{-5}$, the inversion fails to converge. For $\beta$ equal to $10^{-1}$, the reconstructed anomaly is relatively similar to the one produced by the standard smoothness-constraint difference inversion (compare Figure $2 d$ and $2 \mathrm{j}$ ). For the lowest value of the MGS parameter, many artifacts appear at depth (Figure 2f). If we select the $\beta$ value $\left(10^{-4}\right)$ corresponding to the minimum TL data misfit after the first iteration, the result shown in Figure $2 \mathrm{~g}$ is obtained. The latter has much fewer artifacts and reveals a focused anomaly of greater contrast $(-40 \%)$ than the smoothness-constraint TL model changes. Artificial positive resistivity changes remain close to the anomaly with physically reasonable resistivity changes.

\section{CONCLUSIONS}

In this study, we have proposed a data-driven selection procedure to select the MGS parameter value in MGS inversion of TL ERT data. Compared to other approaches, which rely on a choice of the MGS parameter based on prior knowledge or synthetic test cases, our selection strategy does not require prior information and relies on data misfit only. The conducted synthetic study validates the ability of the proposed approach to select the MGS parameter and successfully demonstrates the recovery of a notoriously challenging target. The similarity between the field case and the synthetic study provides confidence in the results obtained by the MGS inversion. Comparison with the standard smoothness-constrained difference inversion results also provides clear indication of the global position and sign of resistivity changes of the anomaly but not its shape.

Our results are dependent on the regularization parameter optimization strategy. The algorithm that we used seeks to fit the data within a certain noise level for a maximum value of the regularization parameter $\lambda$. Not all inversion software and codes are implemented this way. However, the selection of the MGS parameter $\beta$ is made at the first iteration of the inversion; this value is fixed in subsequent iterations, and the inversion results obtained with standard smoothness constraint versus MGS are therefore compared on a fair basis using the same stopping criterion. Although the inverse problem is nonlinear, our hypothesis is that the problem is only weakly nonlinear because we seek for a solution relatively close to the background model. Estimating the MGS parameter $\beta$ during the first iteration can therefore be justified by the TL nature of the inversion. It is also a convenient procedure computationally.

The MGS regularization should be viewed as a fine-tuning tool to further improve observations already obtained with standard inversion approaches, such as the smoothness constraint. The MGS is relatively prone to the creating of artifacts and sensitive to the data weights, i.e., the description of data errors, in the inversion. Future work should focus on further validating the approach for different types of geophysical data, including TL series and more complex changes, and where relatively little prior knowledge is required.
Figure 2. Results from field data. (a) Injection well and setup, (b) nearby outcrop of the fractured limestone, (c) background model (smoothness constraint), (d) inverted TL model changes using smoothness constraint on the model changes, (e) selection of the MGS parameter $\beta$, the corresponding images (f-j) are indicated next to each point. Panels (f-j) show inverted TL model changes using MGS for different values of MGS parameters. Background model values are given as $\log _{10}$ of resistivity in $\Omega \mathrm{m}$ in panel (c), and TL model resistivity changes are given in percentage in panels ( $d$ and $f-j$ ). 


\section{ACKNOWLEDGMENTS}

We would like to thank the associate editors and the three reviewers for providing constructive comments on this paper. This work was supported by the Fonds de la Recherche Scientifique - FNRS under the Grant "Chargé de Recherche" of Thomas Hermans.

\section{REFERENCES}

Ajo-Franklin, J., B. Minsley, and T. Daley, 2007, Applying compactness constraints to differential traveltime tomography: Geophysics, 72 no. 4, R67-R75, doi: 10.1190/1.2742496.

Aster, R. C., B. Borchers, and C. H. Thurber, 2013, Parameter estimation and inverse problems: Elsevier Academic Press.

Belge, M., M. E. Kilmer, and E. L. Miller, 2002, Efficient determination of multiple regularization parameters in a generalized L-curve framework: Inverse Problems, 18, 1161-1183, doi: 10.10.1088/0266-5611/18/4/314.

Blaschek, R., A. Hordt, and A. Kemna, 2008, A new sensitivity-controlled focusing regularization scheme for the inversion of induced polarization data based on the minimum gradient support: Geophysics, 73, no. 2, F45F54, doi: 10.1190/1.2824820.

Brooks, D. H., G. F. Ahmad, R. S. MacLeod, and G. M. Maratos, 1999, Inverse electrocardiography by simultaneous imposition of multiple constraints: IEEE Transactions on Biomedical Engineering, 46, 3-18, doi: 10 $.1109 / 10.736746$.

Day-Lewis, F. D., J. M. Harris, and S. M. Gorelick, 2002, Time-lapse inversion of crosswell radar data: Geophysics, 67, 1740-1752, doi: 10 $.1190 / 1.1527075$

de Groot-Hedlin, C., and S. Constable, 1990, Occam's inversion to generate smooth, two dimensional models from magnetotelluric data: Geophysics, 55, 1613-1624, doi: 10.1190/1.1442813.

Doetsch, J., N. Linde, T. Vogt, A. Binley, and A. G. Green, 2012, Imaging and quantifying salt-tracer transport in a riparian groundwater system by means of 3D ERT monitoring: Geophysics, 77, no. 5, B207-B218, doi: 10 .1190/geo2012-0046.1.

Fiandaca, G., J. Doetsch, G. Vignoli, and E. Auken, 2015, Generalized focusing of time-lapse changes with applications to direct current and time-domain induced polarization inversions: Geophysical Journal International, 203, 1101-1112, doi: 10.1093/gji/ggv350.

Friedel, S., 2003, Resolution, stability and efficiency of resistivity tomography estimated from a generalized inverse approach: Geophysical Journal International, 153, 305-316, doi: 10.1046/j.1365-246X.2003.01890.X.

Hermans, T., S. Wildemeersch, P. Jamin, P. Orban, S. Brouyère, A. Dassargues, and F. Nguyen, 2015, Quantitative temperature monitoring of a heat tracing experiment using cross-borehole ERT: Geothermics, 53, 14-26, doi: 10.1016/j.geothermics.2014.03.013.

Karaoulis, M. C. J. H. Kim, and P. I. Tsourlos, 2011, 4D active time constrained resistivity inversion: Journal of Applied Geophysics, 73, 25-34, doi: 10.1016/j.jappgeo.2010.11.002.

Kemna, A., 2000, Tomographic inversion of complex resistivity: Theory and application: Ph.D. thesis, Ruhr-University of Bochum.

Kemna, A., J. Vanderborght, B. Kulessa, and H. Vereecken, 2002, Imaging and characterisation of subsurface solute transport using electrical resistivity tomography (ERT) and equivalent transport models: Journal of Hydrology, 267, 125-146, doi: 10.10.1016/S0022-1694(02)00145-2.

Kim, J. H., M. J. Yi, S. G. Park, and J. Kim, 2009, 4D inversion of DC resistivity monitoring data acquired over a dynamically changing earth model: Journal of Applied Geophysics, 68, 522-532, doi: 10.1016/j. jappgeo.2009.03.002.

Kim, K. J., and I. K. Cho, 2011, Time-lapse inversion of 2D resistivity monitoring data with a spatially varying cross-model constraint: Journal of Applied Geophysics, 74, 114-122, doi: 10.1016/j.jappgeo.2011.04.010.

Koestel, J., A. Kemna, M. Javaux, A. Binley, and H. Vereecken, 2008, Quantitative imaging of solute transport in an unsaturated and undisturbed soil monolith with 3D ERT and TDR: Water Resources Research, 44, W12411, doi: 10.1029/2007WR006755.

Krautblatter, M., S. Verleysdonk, A. Flores Orozco, and A. Kemna, 2010, Temperature-calibrated imaging of seasonal changes in permafrost rock walls by quantitative electrical resistivity tomography (Zugspitze, German/Austrian Alps): Journal of Geophysical Research, 115, F02003, doi: 10.1029/2008JF001209.

LaBrecque, D. J., M. Miletto, W. Daily, A. Ramirez, and E. Owen, 1996, The effects of noise on Occam's inversion of resistivity tomography data: Geophysics, 61, 538-548, doi: 10.10.1190/1.1443980.

LaBrecque, D. J., and X. Yang, 2001, Difference inversion of ERT data: A fast inversion method for 3-D in situ monitoring: Journal of Environmental and Engineering Geophysics, 6, 83-89, doi: 10.10.4133/JEEG6.2.83.

Miller, C. R., P. S. Routh, T. R. Brosten, and J. P. McNamara, 2008, Application of time-lapse ERT imaging to watershed characterization: Geophysics, 73, no. 3, G7-G17, doi: 10.1190/1.2907156.

Oberdörster, C., J. Vanderborght, A. Kemna, and H. Vereecken, 2010, Investigating preferential flow processes in a forest soil using time domain reflectometry and electrical resistivity tomography: Vadose Zone Journal, 9, 350-361, doi: 10.10.2136/vzj2009.0073.

Ogilvy, R. D., P. I. Meldrum, O. Kuras, P. B. Wilkinson, J. E. Chambers, M. Sen, A. Pulido-Bosch, J. Gisbert, S. Jorreto, I. Frances, and P. Tsourlos 2009, Automated monitoring of coastal aquifers with electrical resistivity tomography: Near Surface Geophysics, 7, 367-375, doi: 10.3997/ 1873-0604.2009027.

Portniaguine, O., and J. Castagna, 2004, Inverse spectral decomposition: 74th Annual International Meeting, SEG, Expanded Abstracts, 17861789.

Portniaguine, O., and M. S. Zhdanov, 1999, Focusing geophysical inversion images: Geophysics, 64, 874-887, doi: 10.1190/1.1444596.

Ramirez, A. L., and R. J. Lytle, 1986, Investigation of fracture flow paths using alterant geophysical tomography: International Journal of Rock Mechanics and Mining Sciences and Geomechanics Abstract, 23, 165-169, doi: 10.1016/0148-9062(86)90343-8.

Robert, T., D. Caterina, J. Deceuster, O. Kaufmann, and F. Nguyen, 2012, A salt tracer test monitored with surface ERT to detect preferential flow and transport paths in fractured/karstified limestones: Geophysics, 77, no. 2 , B55-B67, doi: 10.1190/geo2011-0313.1.

Robert, T., A. Dassargues, S. Brouyère, O. Kaufmann, V. Hallet, and F. Nguyen, 2011, Assessing the contribution of electrical resistivity tomography (ERT) and self-potential (SP) methods for a water well drilling program in fractured/karstified limestones: Journal of Applied Geophysics, 75, 42-53, doi: 10.1016/j.jappgeo.2011.06.008.

Robinson, J., T. Johnson, and L. Slater, 2015, Challenges and opportunities for fractured rock imaging using 3D cross-borehole electrical resistivity: Geophysics, 80, no. 2, E49-E61, doi: 10.1190/geo2014-0138.1.

Rosas Carbajal, M., N. Linde, and T. Kalscheuer, 2012, Focused time-lapse inversion of radio and audio magnetotelluric data: Journal of Applied Geophysics, 84, 29-38, doi: 10.1016/j.jappgeo.2012.05.012.

Singha, K, F. D. Day-Lewis, T. Johnson, and L. D. Slater, 2015, Advances in interpretation of subsurface processes with time-lapse electrical imaging: Hydrological Processes, 29, 1549-1576, doi: 10.1002/hyp.v29.6.

Slater, L. D., D. Ntarlagiannis, F. D. Day-Lewis, K. Mwakanyamale, R. J. Versteeg, A. Ward, C. Strickland, C. D. Johnson, and J. W. Lane, 2010 Use of electrical imaging and distributed temperature sensing methods to characterize surface water-groundwater exchange regulating uranium transport at the Hanford 300 Area, Washington: Water Resources Research, 46, W10533, doi: 10.1029/2010WR009110.

Tikhonov, A. N., and V. A. Arsenin, 1977, Solution of ill-posed problems: Winston \& Sons.

Vignoli, G., G. Fiandaca, A. V. Christiansen, C. Kirkegaard, and E. Auken, 2015, Sharp spatially constrained inversion with applications to transient electromagnetic data: Geophysical Prospecting, 63, 243-255, doi: 10 .1111/1365-2478.12185.

Zhang, L., K. Takao, U. Hisashi, Y. Peng, and W. Jialin, 2012, A regularized three-dimensional magnetotelluric inversion with a minimum gradien support constraint: 3D MT inversion with minimum gradient support: Geophysical Journal International, 189, 296-316, doi: 10.1111/gji .2012.189.issue-1.

Zhdanov, M. S., 2002, Geophysical inverse theory and regularization problems: Elsevier.

Zhdanov, M. S., and E. Tolstaya, 2004, Minimum support nonlinear parametrization in the solution of a 3D magnetotelluric inverse problem: Inverse Problems, 20, 937-952, doi: 10.1088/0266-5611/20/3/017. 